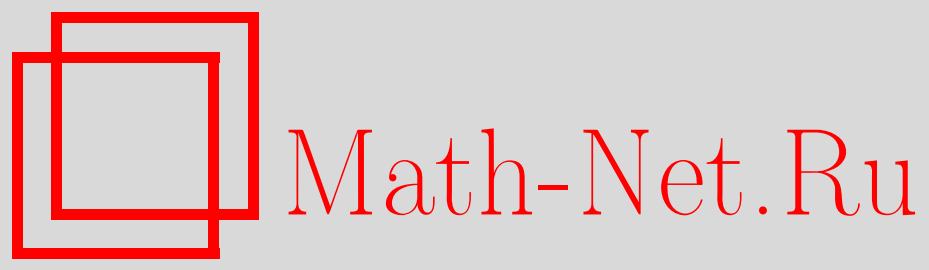

А. Ю. Константинов, Спектральный анализ одного класса матричных дифференциальных операторов, Функи. анализ и его прил., 1997, том 31, выпуск 3, 77-80

DOI: https://doi.org/10.4213/faa481

Использование Общероссийского математического портала MathNet.Ru подразумевает, что вы прочитали и согласны с пользовательским соглашением http://www . mathnet.ru/rus/agreement

Параметры загрузки:

IP : 3.80 .253 .173

26 апреля 2023 г., 13:48:56

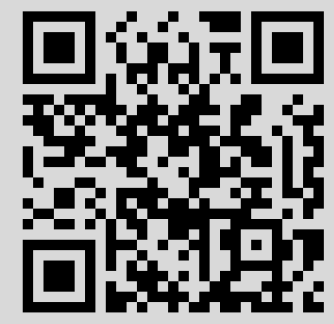




\title{
Спектральный анализ одного класса матричных дифференциальных операторов
}

\author{
(c) 1997. А. Ю. КонСТАнтинов
}

1. В последние годы интенсивно изучаются спектральные свойства рациональных операторнозначных функщий и (или) ассоциированных с ними блочных операторных матриц [1-8]. В частности, с уравнением Шрёдингера с потенциалом, рационально зависящим от спектрального параметра [2-4], ассоциирован оператор вида

$$
\left(\begin{array}{cc}
-\Delta+q & v \\
\bar{v} & h
\end{array}\right)
$$

Операторы типа (1) исследовались в ограниченных областях пространства $\mathbb{R}^{n}$ $[3,4]$. В то же время спектральная теория таких операторов во всем $\mathbb{R}^{n}$ почти не изучена. Отметим лишь недавнюю работу [7], где предлагается абстрактный подход к определению существенного спектра матричных дифференциальных операторов в неограниченной области и ставится вопрос об исследовании более тонкой структуры спектра таких операторов. Настоящая работа посвящена теории рассеяния и спектральному анализу одного класса матричных операторов, включающего в себя операторы вида (1).

2. Рассмотрим в пространстве $\mathscr{H}=\left(L_{2}\left(\mathbb{R}^{n}\right)\right)^{2}$ операторы

$$
H=\left(\begin{array}{cc}
(-\Delta)^{l}+q & \sum_{|\alpha|<l} D^{\alpha} v_{\alpha} \\
\sum_{|\alpha|<l}(-1)^{|\alpha|} \bar{v}_{\alpha} D^{\alpha} & h
\end{array}\right), \quad H_{0}=\left(\begin{array}{cc}
(-\Delta)^{l} & 0 \\
0 & h
\end{array}\right) .
$$

Здесь $l=1,2, \ldots, \alpha \in \mathbb{Z}_{+}^{n}$ - мультииндекс, $D^{\alpha}-$ соответствующая частная производная, $v_{\alpha}$ - ограниченные функции класса $C^{|\alpha|}, q-$ ограниченная и $h$ - непрерывная вещественные функции. Оператор $H$ существенно самосопряжен в $\mathscr{H}$ на $\left(C_{0}^{\infty}\left(\mathbb{R}^{n}\right)\right)^{2}$ (см., например, [5]). Отметим, что при $l=1$ $H$ является оператором вида (1).

ПРЕДЛОЖЕНИЕ 1. Пусть

$$
|q(x)|+\sum_{|\alpha|<l}\left|v_{\alpha}(x)\right|(|h(x)|+1)^{-1} \rightarrow 0, \quad|x| \rightarrow \infty .
$$

Тогда разность резольвент операторов $H$ и $H_{0}$ компактна и, в частноcmu, $\sigma_{\mathrm{ess}}(H)=\sigma_{\mathrm{ess}}\left(H_{0}\right)=[0, \infty) \cup \overline{h\left(\mathbb{R}^{n}\right)}$.

Отметим, что хотя предложение 1 и элементарно, но оно не вытекает из общих результатов [7]. В частности, функции $v_{\alpha}$ могут не убывать на бесконечности. Для изучения более тонких спектральных свойств оператора $H$ мы применяем вариант гладкой теории рассеяния, основанный на локальных коммутаторных оценках Мурра [9-11]. Будем предполагать, что $h \in C^{3}\left(\mathbb{R}^{n}\right)$. Точка $\lambda \in \mathbb{R}$ называется пороговым значением функции $h$, если $\inf \left\{\left|h^{\prime}(x)\right|: h(x) \in J\right\}=0$ для любой открытой окрестности $J$ точки $\lambda$. Ниже $\tau(h)$ - множество пороговых значений функции $h, \tau:=\tau(h) \cup\{0\}, f_{\alpha}:=v_{\alpha}(h+i)^{-1}$. Обозначим через $\sigma_{\text {ас }}(A)$ 
абсолютно непрерывный спектр самосопряженного оператора $A$, а через $P_{\text {ac }}(A)$ проектор на абсолютно непрерывное подпространство этого оператора. Будем говорить, что функция $v$ на $\mathbb{R}^{n}$ является короткодействующим потенциалом (потенциалом Энсса), если

$$
\int_{1}^{\infty} \operatorname{ess} \sup \{|v(x)|:|x| \geqslant r\} d r<\infty .
$$

ТЕОРема 1. Предположим, что

(i) потенциаль $q$ и $f_{\alpha}(|\alpha|<l)$ короткодействуюшие;

(ii) $f_{\alpha} \in C^{2}\left(\mathbb{R}^{n}\right)$, производные $f_{\alpha}^{\prime}, f_{\alpha}^{\prime \prime}$ ограниченьи на $\mathbb{R}^{n} u\left|f_{\alpha}^{\prime}\right|^{2}-\kappa о$ роткодействующий потенциал $(|\alpha|<l)$;

(iii) для произвольного компакта $K \subset \mathbb{R} \backslash \tau$ существует $c>0$, такое, что, как только $h(x) \in K$,

$$
\left|h^{\prime \prime}(x)\right| \leqslant c\left|h^{\prime}(x)\right|^{2}, \quad\left|h^{\prime \prime \prime}(x)\right| \leqslant c\left|h^{\prime}(x)\right|^{3} ;
$$

(iv) множество $\tau$ не более чем счетно.

Тогда

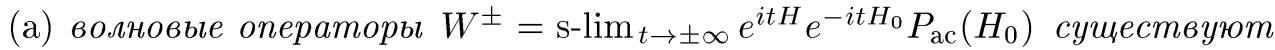
и полньг; в частности, абсолютно непрерьвные части операторов $\mathrm{H}$ и $\mathrm{H}_{0}$ унитарно эквивалентньи и $\sigma_{\mathrm{ac}}(H)=\sigma_{\mathrm{ac}}\left(H_{0}\right)$;

(b) $H$ не имеет сингулярно непрерьвного спектра;

(c) собственнье значения оператора $H$, не принадлежащие $\tau$, конечнократны и не могут иметь (конечных) точек накопления вне $\tau$.

Прокомментируем условия теоремы 1. Пункт (iii) является важным техническим условием, гарантирующим в нашей ситуации возможность применения метода Мурра (см. [10]), а (iv) обеспечивает отсутствие сингулярно непрерывного спектра. Так как невозмущенный оператор $H_{0}$ действует на части пространства $\mathscr{H}$ как степень оператора Лапласа, а на другой части как оператор умножения, то для существования волновых операторов возмущение (разность резольвент операторов $H$ и $H_{0}$ ) должно быть короткодействующим как по пространственным, так и по импульсным переменным. Убывание по пространственным переменным дает условие (i). Убывание по импульсным переменным обеспечивается тем, что порядок возмушения меньше $l$. При этом условие (ii) обеспечивает определенную регулярность возмущения. Оно может быть ослаблено. Например, вместо ограниченности $f_{\alpha}^{\prime \prime}$ достаточно требовать, чтобы операторы умножения на $f_{\alpha}$ были ограничены в соболевском пространстве $W_{2}^{1+\varepsilon}\left(\mathbb{R}^{n}\right)$ при некотором $\varepsilon>0$.

В условиях теоремы 1 для оператора $H$ справедлив принцип предельного поглощения. Пусть $T_{\varepsilon}=\operatorname{diag}\left\{\left(1+|x|^{2}\right)^{-1 / 4-\varepsilon},(-\Delta+1)^{-1 / 4-\varepsilon}\right\}-$ диагональный оператор в $\mathscr{H}$, а $\sigma_{p}(H)$ - точечный спектр оператора $H$.

ТеОРема 2. Пусть выполнены условия (i)-(iii) теоремы 1. Тогда для произвольных $\varepsilon>0$ и компакта $K \subset \mathbb{R} \backslash\left(\tau \cup \sigma_{p}(H)\right)$

$$
\sup \left\{\left\|T_{\varepsilon}(H-z)^{-1} T_{\varepsilon}\right\|_{\mathscr{L}(\mathscr{H})}: \operatorname{Re} z \in K, \operatorname{Im} z \neq 0\right\}<\infty .
$$

Отметим, что в условиях теоремы 2 принцип предельного поглощения выполняется в более тонкой шкале пространств Бесова (см. [10]). 
3. Теорема 1 неверна для возмущений порядка $l$. В этом случае происходит определенный сдвиг существенного спектра оператора $H[7]$ и для пары $H$, $H_{0}$ уже не могут существовать полные волновые операторы. В то же время в связи с различными приложениями в математической физике [12-14] этот случай представляет особый интерес. Здесь мы приведем лишь один модельный пример, показывающий, что техника, развитая при доказательстве теоремы 1, может работать и в этой ситуации. Рассмотрим в пространстве $\mathscr{H}=\left(L_{2}\left(\mathbb{R}^{n}\right)\right)^{n} \times L_{2}\left(\mathbb{R}^{n}\right)$ блочный матричный оператор

$$
H=\left(\begin{array}{cc}
-\Delta+q & -\operatorname{grad} b+v \\
\bar{b} \operatorname{div}+v^{*} & h
\end{array}\right) .
$$

Здесь $h$ - непрерывная вещественная функция на $\mathbb{R}^{n}, b$ - ограниченная гладкая функции на $\mathbb{R}^{n}, q, v$ - ограниченные функции на $\mathbb{R}^{n}$, значениями которых являются соответственно эрмитовы матрицы размера $n \times n$ и комплексные матрицы размера $n \times 1, v^{*}$ - матрица, сопряженная к $v$. В этих предположениях $H$ существенно самосопряжен на $\left(C_{0}^{\infty}\left(\mathbb{R}^{n}\right)\right)^{n+1}$ (см., например, [5]). Положим $f:=(h+i)^{-1} v, g:=(h+i)^{-1} b, h_{0}:=h-|b|^{2}$.

ПРЕДЛОЖЕНИЕ 2. Предположим, что

$$
|q(x)|+|g(x)|+|f(x)| \rightarrow 0, \quad|x| \rightarrow \infty .
$$

Тогда $\sigma_{\text {ess }}(H)=[0, \infty) \cup \overline{h_{0}\left(\mathbb{R}^{n}\right)}$.

Отметим, что для убывающих на бесконечности функций $b$ и $v$ предложение 2 вытекает из результатов [7]. Ниже считаем, что $h_{0} \in C^{4}\left(\mathbb{R}^{n}\right)$ и $\tau=\tau\left(h_{0}\right) \cup\{0\}$.

ТЕОРемА 3. Предположим, что

(i) $q, f, g$ - короткодействующие потенциальг;

(ii) $f, g \in C^{2}\left(\mathbb{R}^{n}\right)$ и функиии $f^{\prime}, f^{\prime \prime}, g^{\prime}, g^{\prime \prime}$ ограниченьл;

(iii) для некоторого $c>0$ и всех $x \in \mathbb{R}^{n}$

$$
\left|h_{0}^{\prime \prime}(x)\right|+\left|h_{0}^{\prime \prime \prime}(x)\right|+\left|h_{0}^{\mathrm{IV}}(x)\right| \leqslant c\left(1+\left|h_{0}(x)\right|+\left|h_{0}^{\prime}(x)\right|\right) ;
$$

(iv) множество $\tau\left(h_{0}\right)$ не более чем счетно.

Тогда $\sigma_{\mathrm{ac}}(H)=[0, \infty) \cup \sigma_{\mathrm{ac}}\left(h_{0}\right)$ и выполнень утверждения (b), (c) теоремь 1 и утверждения теоремь 2.

Предложение 2 и теорема 3 показывают, что для убывающих функций $q, g, f$ оператор $H$ естественно рассматривать как возмущение «сдвинутого» диагонального оператора $H^{0}=\operatorname{diag}\left\{-\Delta, h_{0}\right\}$. В то же время это возмущение уже не является короткодействующим по импульсной переменной $p$ (разность резольвент операторов $H$ и $H^{0}$ убывает, как $\left.|p|^{-1}\right)$. Последнее не позволяет, вообще говоря, утверждать существование обычных волновых операторов для пары $H$ и $H^{0}$ (cp. с теоремой 1$)$.

\section{ЛИТЕРАТУРА}

1. Adamjan V. M., Langer H. J. Operator Theory, 33, 259-277 (1995). 2. Богомолова Е. П. Диф. уравн., 21, №11, 1843-1849 (1985). 3. Faierman M., Mennicken R. Manuscripta Mathematica, 73, 319-333 (1991). 4. Langer H., Mennicken R., Möller M. Operator Theory: Advances and Applications, 48, 319-332 (1990). 5. Mennicken R., Shkalikov A. A. Math. Nachr., 179, 259-273 (1996). 6. Мотовилов А. К. ТМФ, 104, №2, 281-303 (1995). 7. Шкаликов A. A. Матем. заметки, 58, №6, 945-949 
(1995). 8. Степин C. A. Функц. анализ и его прил., 29, вып. 2, $22-35$ (1995). 9. Mourre E. Comm. Math. Phys., 78, 391-408 (1981). 10. Amrein W., Boutet de Monvel A., Georgescu V. $C_{0}$-Groups, Commutator Methods and Spectral Theory of $N$-Body Hamiltonians. Birkhäuser, Basel (1996). 11. Цикон Х., Фрезе Р., Кири В., Саймон Б. Операторы Шрёдингера с приложениями к квантовой механике и глобальной геометрии. Мир, М. (1990). 12. Ландау Л. Д., Лифиии Е. М. Гидромеханика. Наука, М. (1986). 13. Lifschitz A. E. Magnetohydrodinamics and spectral theory. Kluwer Acad. Publishers, Dodrecht (1989). 14. Копачевский Н. Д., Крейн С. Г., Нго Зуй Кан. Операторные методы в линейной гидродинамике. Наука, М. (1989).

Киевский университет,

кафедра математического анализа

Поступило в редакцию 28 июля 1996 г.

УДК 517.98

\section{О почти коммутирующих операторах *}

(c) 1997. В. М. МАнУйлов

В настоящей заметке исследуется вопрос о возможности прогомотопировать один из двух почти коммутирующих операторов к единице так, чтобы норма коммутатора вдоль всего пути была мала. В общем случае ответ отрицательный $[4,2]$, более того, в работе [2] определено топологическое препятствие для существования такой гомотопии. Мы приводим случай, когда такая гомотопия существует. Напомним, что условие $R R(A)=0$ означает [1], что среди самосопряженных элементов $C^{*}$-алгебры $A$ плотны элементы с конечным спектром; поэтому без ограничения общности можно считать, что $h=\sum_{i=1}^{n} \lambda_{i} p_{i}-$ конечная линейная комбинация проекторов $p_{i} \in A$, где собственные значения упорядочены по убыванию. Напомним также, что условие $\operatorname{tsr}(A)=1$ означает [3], что обратимые элементы плотны в $A$.

Tеорема 1. Пусть $A$ есть $C^{*}$-алгебра со следуюшими свойствами:

(i) $R R(A)=0 \quad u \operatorname{tsr}(A)=1$;

(ii) для любого проектора $p \in A$ унитарная группа $C^{*}$-алгебры $p A p$ свлзна. Пусть $h, u \in A-$ самосопряженный и унитарньй элементы, такие, что

$$
\left\|u^{*} h u-h\right\|<\delta .
$$

Тогда существует константа $C$, зависящал только от $\|h\|$, и путь $u(t)$, соединяющий и с 1 , такой, что при достаточно малом $\delta$ для всех $t$

$$
\left\|u^{*}(t) h u(t)-h\right\|<C \sqrt[4]{\delta} .
$$

ДоказАтельство. Разобьем спектр оператора $h$ на мелкие отрезки длины $\sqrt[4]{\delta}$ и пронумеруем те из них, которые содержат хотя бы одну точку спектра. Тогда $\operatorname{Sp}(h) \subset \bigcup_{k=1}^{m} \Delta_{k}$. Без ограничения общности можно считать, что точки спектра оператора $h$ не совпадают с концами отрезков $\Delta_{k}$. Отметим, что если отрезки $\Delta_{k}$ и $\Delta_{k+1}$ не примыкают друг к другу, то спектр оператора $h$ имеет лакуну длины не менее чем $\sqrt[4]{\delta}$. Обозначим спектральные проекторы оператора $h$, отвечающие отрезкам $\Delta_{k}$, через $q_{k}, q_{1}+\cdots+q_{m}=1$.

* Настоящая работа выполнена при частичной поддержке РФФИ (грант №96-01-00182). 